\title{
Minimum GPRS Bandwidth for Acceptable H.261 Video QoS
}

\author{
Iyad Al Khatib, Anders Franzen, Fabio Moioli \\ Department of Teleinformatics, The Royal Institute of Technology \\ Ericsson Business Networks, and Ericsson Wireless LAN Systems, Sweden \\ iyad@it.kth.se, \{Anders.franzen, Fabio.moioli\}@era.ericsson.se
}

Key words: GPRS, H.261 video codec, Hurst parameter, multiplexing gain

Abstract: As part of a larger research on multimedia traffic performance over GPRS, we present a QoS study focusing on one parameter: bandwidth. GPRS is an evolutionary phase and a critical step towards the third generation (3G) of mobile systems providing a data rate of $2 \mathrm{Mbps}$. In our study on GPRS, we investigate multimedia video traffic. The video codec used is $\mathrm{H} .261$ with QCIF resolution. Many parameters are under research; however, in this paper we focus on one parameter: minimum required bandwidth for acceptable QoS of QCIF H.261 video streams over the wireless and mobile medium, GPRS. Some other parameters of interest like the Hurst parameter and multiplexing gain are tackled.

\section{INTRODUCTION}

The wireless and mobile telecommunication world is experiencing a very critical transitional stage, where new QoS parameters are to be defined. Within a more general study on mobile systems evolution, we analyse multimedia traffic over GPRS, a new phase of mobile communication media. GPRS represents an evolutionary step from the existing GSM system, where its purpose is to bring packet switched data services to the mobile system. With GPRS, the user can always be connected to the network since charging is not based on the connection time. The final billing scheme is not totally defined yet, but the main point is that the user should not care aboutconnection time. 
One of the other goals of GPRS is to try to provide higher speeds than traditional GSM systems. The maximum theoretical speed over GPRS is supposed to be around $115 \mathrm{Kbps}$. This bandwidth is achieved with very good radio conditions, and when the network is fully developed. In practice, the starting GPRS speed would, to a large probability, be somewhere between $20 \mathrm{Kbps}$ and 56Kbps. An enhanced GPRS system called EDGE is supposed to bring the speed up to $384 \mathrm{Kbps}$. This very evolutionary phase of mobile systems is believed to be only one step towards the third mobile systems generation ( $3 \mathrm{G})$, which is expected to give speeds up to $2 \mathrm{Mbps}$.

The GSM system uses Time-Division Multiple Access (TDMA) with eight radio frequency time slots. A network operator can dedicate 0 to 8 of these time slots to GPRS. Each mobile terminal can send/receive in 1 to 8 time slots. It is believed that the first mobile terminals generation for GPRS will support 4 time slots downlink and 1 time slot uplink, which gives around $14 \mathrm{Kbps}$ uplink and 56Kbps downlink.

With this great shift that GPRS will introduce to the wireless and mobile world, we are interested in investigating the quality of service that GPRS can offer to multimedia applications, mainly video quality. Our research in this area is long term; however, in this paper we investigate few multimedia traffic parameters for one video standard. The format of the video streams we investigate over GPRS is H.261 with QCIF resolution. H.261 is chosen for its low bit rate [10]. The H.261 video streams in the experiments are variable bit rate streams; which makes them more suitable for the medium [4]. Quarter-CIF (QCIF) has 176 pixels per line, and 144 lines [9]. QCIF is chosen, because it is mainly used for desktop videophone applications i.e. the size will be suitable for a mobile unit. In addition, all codecs must be able to handle QCIF.

The parameter we focus on throughout the experiments is the minimum GPRS bandwidth required for acceptable QoS of H.261 video streams of QCIF resolution. We are also interested in self similarity since if we can define which type of videos show self similarity over GPRS, then GPRS vendors can learn more about how to deal with video over this medium $[3,6]$. In this respect, the Hurst parameter is calculated. The Hurst parameter can be looked at as a self-similarity value; if near to 1 , then this would be a sign of self-similarity. However, if it shows a value nearer to 0.5 , then there is not much of self-similarity in the traffic.

In a study of multimedia over GPRS, it is very important to note that the standards with which the QoS is judged are subjective. Unfortunately, up till now, the judgements on acceptable QoS for multimedia streams are relative to the observer's personal standards [8]. Hence, we find it very important that, in our study, the minimum acceptable parameters investigated are defined by a representative number of people from different 
population backgrounds. Hence a common acceptable QoS is set to find the minimum bandwidth sought.

To calculate the theoretical values for the minimum acceptable bandwidth, we use the Multiplexing Gain formula [5]:

$$
G_{n}=n R_{p} / C_{n}
$$

where $R_{p}$ is peak rate for the video stream; $n$ is the number of independent streams combined for transmission; and $C_{n}$ is the link-bandwidth required for the desired QoS for the multiplexed stream of $n$ sources $\left(\mathrm{C}_{1}\right.$ being the link bandwidth for a single source).

$$
(1)=>G_{n}=n R_{p} / C_{n}=\left[n C_{1} / C_{n}\right]\left[R_{p} / C_{l}\right]=\left[n C_{l} / C_{n}\right] G_{1}
$$

where $G_{l}$ is the multiplexing gain for one source.

$$
\text { (2) } \Leftrightarrow C_{n}=n\left[G_{1} / G_{n}\right] C_{l}
$$

Here we think of the multiplexing gain as a parameter to use in order to achieve the minimum link-bandwidth for $n$ streams where $n \in N^{*}$, the set of natural numbers - $\{0\}$. The multiplexing gain $G_{n}$ for $n$ number of independent streams is given by,

$$
\frac{1}{G_{n}}=\frac{1}{b}+\left(\frac{1}{G_{1}}-\frac{1}{b}\right) n^{\frac{1-2 H}{2 H}}
$$

where $b$ is the peak-to-average and $H$ is the Hurst parameter. Many methods can be used to calculate the Hurst parameter, like time variance plot, R/S analysis [2], and periodogram method.

\section{EXPERIMENTS AND RESULTS}

Figure 1 shows the testbed, which consists of two video senders, a GPRS emulator, a receiver, and a traffic measurement and analysis tool, NIKSUN NetVCR ${ }^{\mathrm{TM}}$. All the experiments, except the last one, use one sender only, for they are dedicated to studying the bandwidth required for one video stream. On the other hand, the last experiment concentrates on the performance when multiple streams are sent over GPRS. Table 1 shows the video streams, where "Comm" is the stream used in experiments 1 to 5. The packet time slots on the GPRS medium are set to 8 time slots throughout all the experiments since using less for video transmission will not lead to acceptable QoS. First, we look into whether there is any difference between 
the behavior of two media: GPRS with no restricting limits, and 10-BT. The associated results for the H.261 video stream are presented in table 2, where one would conclude that when the GPRS is dedicated to one video stream, with no background users, it will most likely behave like Ethernet. However, when running the first experiment on $10 \mathrm{Mbps}$ Ethernet, we got no missing frames at the receiver end, while in running the experiment over GPRS, with $12 \mathrm{~dB}$, we had 2,670 video frames missing out of 6,306 video frames of the same stream (see table 4, Exp. 1 and 2). Figures 1-8 show the number of bytes (vertical axis) versus the packet size categories (horizontal axis).

Table 1. Video sequences used in the experiments. "Comm" is used in experiments 1 to 5.

\begin{tabular}{|l|c|c|c|c|c|}
\hline $\begin{array}{c}\text { Type of } \\
\text { video }\end{array}$ & $\begin{array}{c}\text { Length } \\
\text { (mm:ss) }\end{array}$ & Total bytes & $\begin{array}{c}\text { Total } \\
\text { packets }\end{array}$ & $\begin{array}{c}\text { Average } \\
\text { bandwidth (bps) }\end{array}$ & $\begin{array}{c}\text { Hurst } \\
\text { param. }\end{array}$ \\
\hline Music 1 & $05: 18$ & $1,769,912$ & 7,941 & 44,387 & 0.81 \\
\hline Music 2 & $03: 39$ & $3,027,270$ & 4,745 & 109,584 & 0.78 \\
\hline Music 3 & $03: 25$ & $2,633,906$ & 4,582 & 101,793 & 0.88 \\
\hline News & $13: 55$ & $10,657,756$ & 18,313 & 101,623 & 0.74 \\
\hline Talking head & $12: 51$ & $11,682,038$ & 13,682 & 120,901 & 0.61 \\
\hline $\begin{array}{c}\text { Commercial } \\
\text { "Comm" }\end{array}$ & $05: 06$ & $3,631,412$ & 6,942 & 94,322 & 0.79 \\
\hline \multicolumn{1}{|c|}{ Total } & $44: 14$ & $33,402,294$ & 56,205 & $\ldots$ & \\
\hline
\end{tabular}

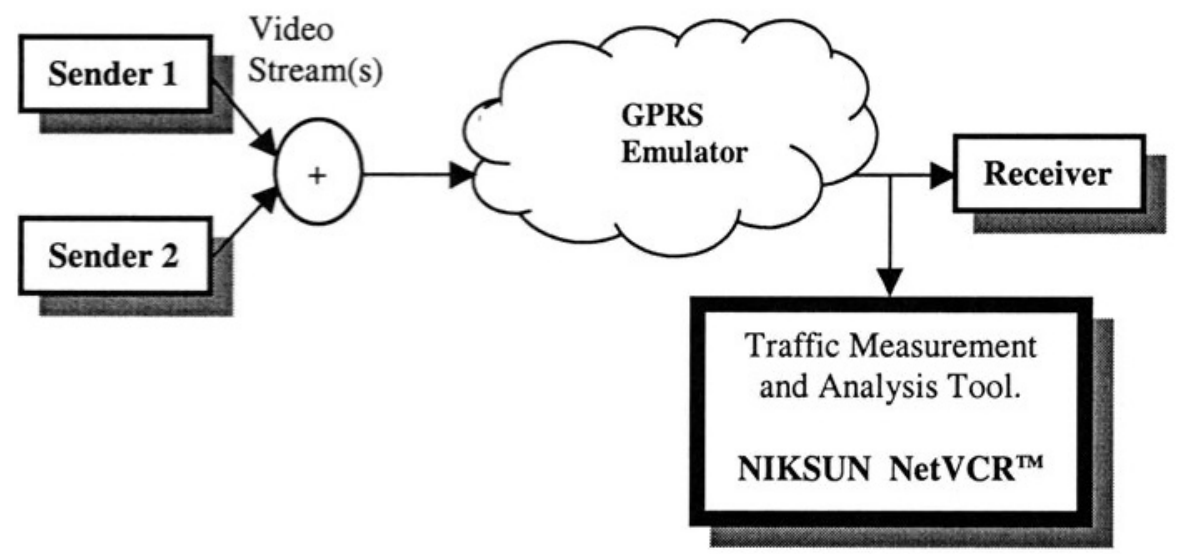

Figure 1. Testbed of bandwidth investigation for $H .261$ video quality over GPRS.

Hence in figure 1, the peak (bytes) is for the packets that are 5121024 bytes in size, excluding 1024 byte packets. The second level peaks are for packets of 1024-2048 bytes (excluding 2048 byte packets) and 216-512 bytes (excluding 512 byte packets) respectively. Figures 2 through 8 can be read in a similar way for the associated experiments. 
Table 2. Differences between GPRS with no restrictions and 1OBT for "Comm". S/N=12 dB.

\begin{tabular}{|c|c|c|}
\hline & No limits on GPRS & 10 Mbps Ethernet \\
\hline Total no. of bytes received & $1.7284 \mathrm{e}+003$ & $1.7289 \mathrm{e}+003$ \\
\hline Median & 3848 & 3788 \\
\hline Peak-to-Average ratio & 5.3804 & 4.7394 \\
\hline
\end{tabular}

We use the parameters in tables 3 and 4 to do some calculations for comparisons with the values received by the application. In this respect, we would like to note that the video is observed in real time and then the number of missing video frames is calculated. We believe that these numbers are very important to relate to acceptable QoS of the H.261 streams over GPRS. The fact that no frame is missing in experiment 1 can also be seen while watching the video stream live. Experiment 1 is also used as a comparison basis for acceptable QoS.

Table 3. Number of packets vs packet size for "Comm" video stream.

\begin{tabular}{|c|c|c|c|c|c|}
\hline \multirow{2}{*}{$\begin{array}{c}\text { Packet Size } \\
\text { Categories }\end{array}$} & \multicolumn{5}{|c|}{ Count (Packets) } \\
\cline { 2 - 6 } (Bytes) & Exp.1,fig.1 & $\begin{array}{c}\text { Exp.2,fig.2 } \\
12 \mathrm{~dB} \\
\text { 0 BGU }\end{array}$ & $\begin{array}{c}\text { Exp. 3,fig.3 } \\
15 \mathrm{~dB} \\
\text { 0 BGU }\end{array}$ & $\begin{array}{c}\text { Exp. 4,fig.4 } \\
12 \mathrm{~dB} \\
20 \mathrm{BGU}\end{array}$ & $\begin{array}{c}\text { Exp.5,fig.5 } \\
12 \mathrm{~dB} \\
40 \mathrm{BGU}\end{array}$ \\
\hline 0 to 128 & 507 & 305 & 271 & 282 & 275 \\
\hline 128 to 256 & 1089 & 651 & 594 & 585 & 623 \\
\hline 256 to 512 & 2332 & 1332 & 1365 & 1302 & 1367 \\
\hline 512 to 1024 & 2152 & 1312 & 1303 & 1232 & 1302 \\
\hline 1024 to 2048 & 862 & 658 & 672 & 606 & 641 \\
\hline 2048 & 0 & 14 & 19 & 12 & 21 \\
\hline
\end{tabular}

Table 4. Statistics for "Comm" Video Stream over 10 Base-T and GPRS.

\begin{tabular}{|c|c|c|c|c|c|}
\hline & \multirow{2}{*}{$\frac{10 \mathrm{BT}}{\text { Exp. } 1}$} & \multicolumn{4}{|c|}{ GPRS } \\
\hline & & $\begin{array}{l}\operatorname{Exp.2} \\
12 \mathrm{~dB} \\
0 \mathrm{BGU}\end{array}$ & $\begin{array}{l}\text { Exp. 3 } \\
15 \mathrm{~dB} \\
0 \mathrm{BGU}\end{array}$ & $\begin{array}{c}\text { Exp. } 4 \\
12 \mathrm{~dB} \\
20 \mathrm{BGU}\end{array}$ & $\begin{array}{c}\text { Exp. 5 } \\
12 \mathrm{~dB} \\
40 \mathrm{BGU} \\
\end{array}$ \\
\hline $\begin{array}{l}\text { Total Number of } \\
\text { Bytes }\end{array}$ & 7262824 & 4639074 & 4674424 & 4366946 & 4614556 \\
\hline Average Rate (bps) & 48418.83 & 77317.90 & 103876.09 & 72782.43 & 102545.69 \\
\hline Number of Packets & 13884 & 8544 & 8448 & 8038 & 8458 \\
\hline Average Rate (pps) & 11.57 & 17.80 & 23.47 & 16.75 & 23.49 \\
\hline Minimum Packet Size & 67 & 69 & 68 & 67 & 69 \\
\hline $\begin{array}{l}\text { Maximum Packet } \\
\text { Size }\end{array}$ & 1066 & 1066 & 1066 & 1066 & 1066 \\
\hline Mean Packet Size & 523.11 & 542.96 & 553.32 & 543.29 & 545.58 \\
\hline $\begin{array}{l}\text { Packet Size Variance, } \\
\mathrm{B}^{2}\end{array}$ & 93519.15 & 101585.97 & 100581.10 & 99668.34 & 99399.37 \\
\hline Variance/Mean & 178.78 & 187.10 & 181.78 & 183.45 & 182.19 \\
\hline $\begin{array}{l}\text { Missing Video } \\
\text { Frames }\end{array}$ & 0 & 2670 & 2719 & 2811 & 2788 \\
\hline
\end{tabular}


In the second experiment GPRS is used. The $\mathrm{S} / \mathrm{N}$ is $12 \mathrm{~dB}$ i.e. worst case. However the main concern of this experiment is to measure the same parameters as in experiment 1 but over GPRS, hence we have no BackGround Users (BGU) i.e. the "Comm" video has all the bandwidth. The results are presented in figure 2. Collecting these parameters, we can also find a great similarity in the QoS delivered as well as the shapes of the graphs in figures 1 and 2 . Around $41 \%$ of the frames are missing, but still the QoS is acceptable. The receiver end shows a rate ranging between $48 \mathrm{Kbps}$ and $62 \mathrm{Kbps}$, which is a very good rate in our point of view for a video transmission with a $\mathrm{QCIF}$ resolution.

We also investigate the behavior and the used-bandwidth results for the $15 \mathrm{~dB} \mathrm{~S} / \mathrm{N}$. We run exactly the same experiment as in experiment 2 , but with $15 \mathrm{~dB}$ instead of $12 \mathrm{~dB}$. The result is shown in figure 3. This experiment shows just a slight difference where there are more of large packets i.e. the traffic concentration is more in the middle (512-1024B) than in experiment 2. The rate ranges between $48 \mathrm{Kbps}$ and $63 \mathrm{Kbps}$ i.e. acceptable.

To get more practical results, we force background users over the GPRS network. The number of background users we have in experiment 3 counts to 20 , with $12 \mathrm{~dB}$. The result can be seen in figure 4 . The behavior still shows a graph similar to the previous experiments. The rate at the receiver's end still shows a range between $40 \mathrm{Kbps}$ and $60 \mathrm{Kbps}$.

In the fifth experiment we force 40 users with $12 \mathrm{~dB}$ over GPRS. The behavior is similar to the previous experiments in terms of graphical shape (figure 5), but the video quality drops down. In fact it is not acceptable at all. However, the rate at the receiver's end still shows a range between $48 \mathrm{Kbps}$ and $60 \mathrm{Kbps}$.

In the sixth experiment our concentration is on the bandwidth when multiple streams are injected over GPRS, $12 \mathrm{~dB}$ and $40 \mathrm{BGU}$. The results are predictable as shown in figures 6,7 and 8. Filtering the traffic of each stream alone is important to study the bandwidth from the multiplexing gain point of view. Figure 6 shows the result for the traffic of both H.261 video streams at the same time. Since both streams, when injected together, have their first level peaks at (512-1024B), as well as their second level peaks at the same points (figures 7 and 8), then adding the two would lead to a graph with peaks at the same relative points (figure 6). For the first stream, the rate at the receiver's end still shows a range between $21 \mathrm{Kbps}$ and $37 \mathrm{Kbps}$. For the second stream, the rate at the receiver's end still shows a range between $30 \mathrm{Kbps}$ and $50 \mathrm{Kbps}$. The rates show that the bandwidth is divided, and this is a normal behavior. Around $60 \%$ of the frames are lost in each video stream. The visual effects on the QoS can be observed, and the delay between the frames is not within the acceptable range when there are transitions in the video stream. We also run multiple streams over GPRS to 
investigate more on the multiplexing gain and suitable bandwidth for the set QoS. Results are shown in table 7.

Referring to equation 3 , if we know $G_{l}$ and $C_{I}$, then knowing $C_{n}$ will be just a matter of knowing $G_{n}$, which can be calculated using equation 4 . For an acceptable QoS, we will use equation 1 to get to a $C_{l}=R_{p}$, where $R_{p}$ is investigated in the experiments to be around $70 \mathrm{Kbps}$. This makes $G_{l}=1$ for acceptable QoS. Hence (3) becomes: $C_{n}=n\left(1 / G_{n}\right)(70)=70 n\left(1 / G_{n}\right)$.

Table 5. Number of packets vs packet size; 2 video streams; GPRS, $12 d B, 40 B G U$.

\begin{tabular}{|c|c|c|c|}
\hline \multirow{3}{*}{$\begin{array}{c}\text { Packet Size } \\
\text { Categories } \\
\text { (Bytes) }\end{array}$} & \multicolumn{3}{|c|}{ Count (Packets) } \\
\hline & \multicolumn{3}{|c|}{$G \overline{P R S}, 12 \mathrm{~d} B, 40 \mathrm{~B} G U$} \\
\hline & $\begin{array}{c}\text { Total of Two video } \\
\text { streams }\end{array}$ & $\begin{array}{c}\text { First video stream } \\
\text { filtered }\end{array}$ & $\begin{array}{c}\text { Second video stream } \\
\text { filtered }\end{array}$ \\
\hline 0 to 64 & 355 & - & - \\
\hline 64 to 128 & 307 & 162 & 144 \\
\hline 128 to 256 & 824 & 366 & 313 \\
\hline 256 to 512 & 1433 & 747 & 699 \\
\hline 512 to 1024 & 1452 & $\overline{730}$ & $7 \overline{28}$ \\
\hline 1024 to 2048 & 624 & 304 & 305 \\
\hline 2048 & 13 & 0 & 8 \\
\hline
\end{tabular}

Table 6. Two video streams over GPRS, $12 d B, 40 B G U$.

\begin{tabular}{|c|c|c|c|}
\hline & \multicolumn{3}{|c|}{$G P R S, 12 d B, 40 B G U$} \\
\hline & $\begin{array}{l}\text { Total of Two } \\
\text { video streams }\end{array}$ & $\begin{array}{c}\text { First video stream } \\
\text { filtered }\end{array}$ & $\begin{array}{l}\text { Second video } \\
\text { stream filtered }\end{array}$ \\
\hline Total number of Bytes & 4949190 & 2456798 & 2401096 \\
\hline Average Rate (bps) & 94270.27 & 16378.65 & 45735.16 \\
\hline Number of Packets & 10016 & 4618 & 4394 \\
\hline Average Rate (pps) & 23.85 & 3.85 & 10.46 \\
\hline Minimum Packet Size & 60 & 72 & 68 \\
\hline Maximum packet size & 1066 & 1066 & 1066 \\
\hline Mean Packet size & 494.13 & 532.00 & 546.45 \\
\hline Packet Size Variance, $\mathrm{B}^{2}$ & 10533.95 & 96162.32 & 97318.30 \\
\hline Variance/Mean & 213.17 & 180.75 & 178.09 \\
\hline Missing video frames & & 4499 & 4399 \\
\hline
\end{tabular}

Table 7. Multiplexing gain and min. bandwidth for a increasing number of video streams.

\begin{tabular}{|c|c|c|c|c|c|c|c|}
\hline $\begin{array}{c}\text { No. of } \\
\text { Streams }\end{array}$ & $\begin{array}{c}\text { Average } \\
\text { (bits/interval) }\end{array}$ & $\begin{array}{c}\text { Peak } \\
\text { (bits) }\end{array}$ & $\begin{array}{c}\text { Peak-to- } \\
\text { Average }\end{array}$ & $\begin{array}{c}\text { Hurst } \\
\text { Param. }\end{array}$ & Mux Gain & $\begin{array}{c}\text { Minimum } \\
\text { Bandwidth }\end{array}$ \\
\hline 2 & 198,307 & 278,528 & 1.40 & 0.87 & 1.82 & $\Rightarrow>$ & $76.9 \mathrm{Kbps}$ \\
\hline 3 & 297,458 & 455,384 & 1.53 & 0.79 & 1.83 & $\Rightarrow$ & $114.8 \mathrm{Kbps}$ \\
\hline 5 & 487,719 & 628,664 & 1.29 & 0.82 & 1.60 & $\Rightarrow>$ & $218.8 \mathrm{Kbps}$ \\
\hline 10 & 970,794 & $1,246,264$ & 1.28 & 0.83 & 1.50 & $\Rightarrow>$ & $466.7 \mathrm{Kbps}$ \\
\hline 15 & $1,455,795$ & $2,002,776$ & 1.38 & 0.84 & 1.54 & $\Rightarrow>$ & $681.8 \mathrm{Kbps}$ \\
\hline
\end{tabular}




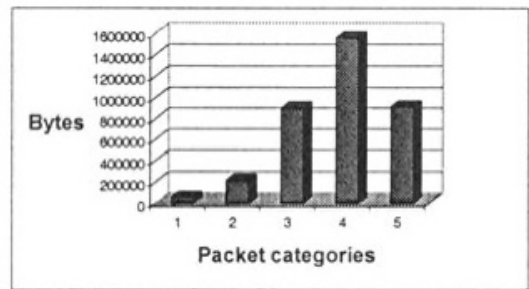

Figure 1. Exp. 1, "Comm" bytes vs packet categories over 10-BT.

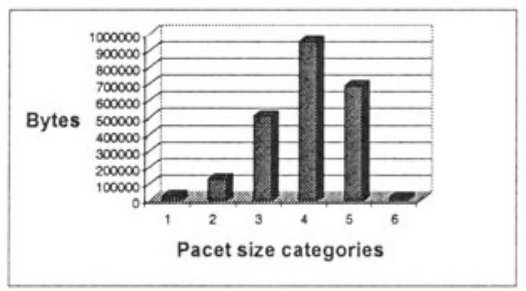

Figure 2. Exp. 2, "Comm" bytes vs packet size categories; GPRS, I2dB, $O B G U$.

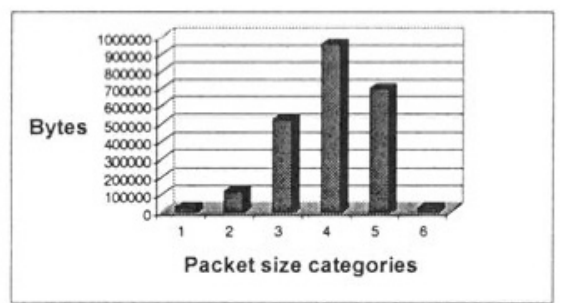

Figure 3. Exp. 3, "Comm" bytes vs packet size categories; GPRS, 15dB, OBGU.

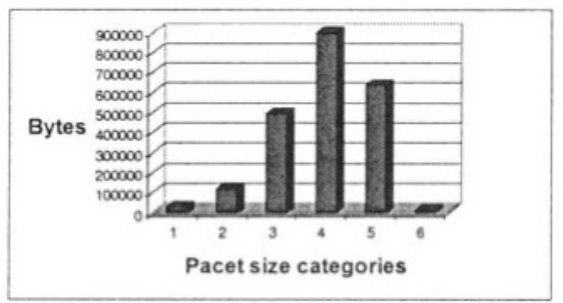

Figure 4. Exp. 4, "Comm" bytes vs packet size categories; GPRS, $12 \mathrm{~dB}, 20 \mathrm{BGU}$.

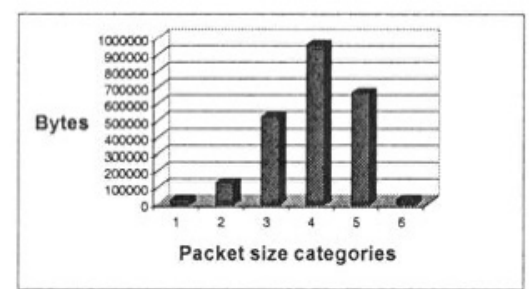

Figure 5. Exp. 5, "Comm" bytes vs packet size categories; GPRS, $12 d B, 40 B G U$.

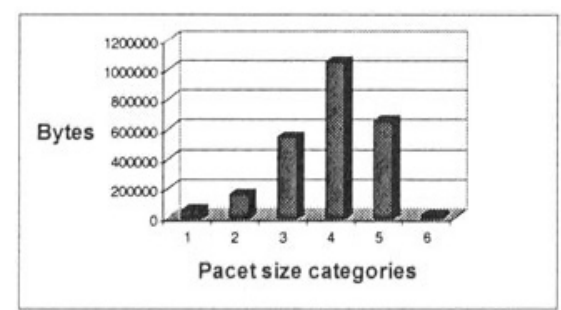

Figure 6. Exp. 6, two video streams traffic over GPRS, $12 d B, 40 \mathrm{BGU}$.

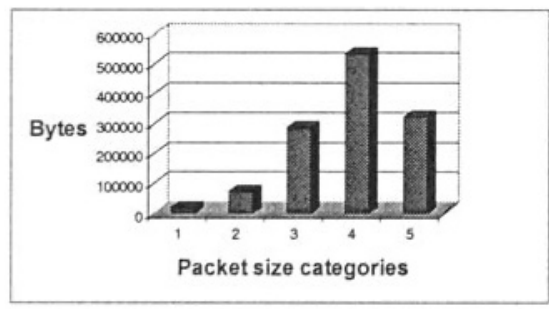

Figure 7. Exp. 6, first video stream over GPRS, I2dB, $40 B G U$.

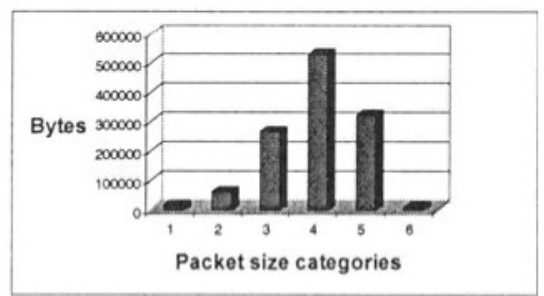

Figure 8. Exp. 6, second video stream over GPRS, 12dB, $40 \mathrm{BGU}$.

Figures 1-8. Horizontal axes show packet size categories of size $X$ bytes, where labels 1, 2 , $3,4,5$, and 6 represent categories with $I \leq X<128$ bytes, $128 \leq X<256$ bytes, $256 \leq X<$ 512 bytes, $512 \leq X<1024$ bytes, $1024 \leq X<2048$ bytes, and $X=2048$ bytes respectively. 


\section{EVALUATION OF RESULTS}

As we increase the number of users and limitations on the GPRS, our calculations lead to a final value that we would like to present. The minimum acceptable bandwidth for H.261 video streams QoS over GPRS is found - after many iterations and trials - to be around 70-80Kbps for one QCIF H.261 video stream. This number is not very satisfactory since the practical limit that GPRS can deliver now is around 50Kbps. However, work is going on to reach higher practical limits, and if $70 \mathrm{Kbps}$ is reached, then sending video streams with QCIF resolution will be possible for the defined QoS. When a bandwidth of less than $70 \mathrm{Kbps}$ over GPRS is reached, the video quality and the missing frames number are not acceptable. In this respect, and regarding the transmission of multiple streams to one receiver to two different application port numbers, the sharing of the bandwidth will surely happen. However, the results in table 7 clearly show that the bandwidth needed over GPRS for the H.261 video for $(n)$ streams will be less than the sum of the peak rates of the two streams. In other words, multiplexing gain will occur and will be a value greater than 1 ;

$$
C_{n}=n R_{p} / G_{n}<n R_{p}=n C_{l} ; \quad G_{n}>1, n \in N^{*} .
$$

The quality with multiple streams will always be less than for one video sent as shown in experiment 6 .

One parameter that seems promising for more research is the Hurst parameter shown in figure 9 with a Log variance vs Log lag plot. Since the self similarity is an interesting parameter to look at when all the presented data is available [7], we look at the Hurst parameter for two streams. The two H.261 video streams over GPRS show a Hurst parameter of around 0.97, with $12 \mathrm{~dB}, 40 \mathrm{BGU}$. This means that the self-similarity is highly probable to occur [1]. This still needs more study to be conducted, but it is a very interesting start.

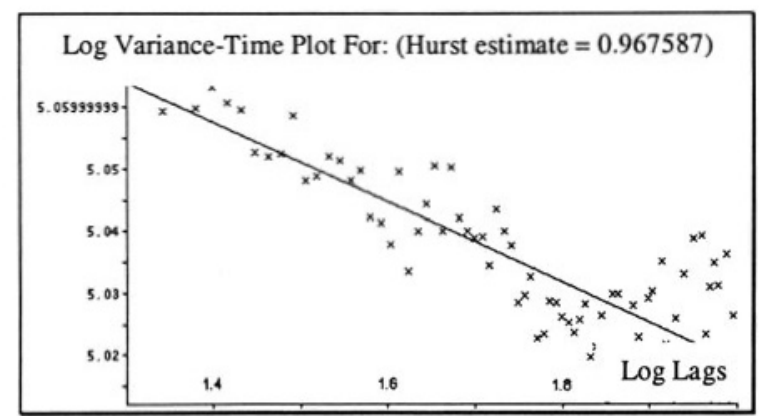

Figure 9. Hurst parameter plot for 2 video streams over GPRS, $12 \mathrm{~dB}$ and $40 \mathrm{BGU}$. 


\section{CONCLUSION}

We investigate some multimedia traffic parameters over GPRS, the third generation of mobile systems. The video streams investigated are encoded in H.261 codec. QCIF resolution is chosen for investigation since it can be deployed on mobile units. The minimum bandwidth required for acceptable QoS of QCIF H.261 video is dependent on the peak rate of the video, the number of streams, and how much the medium can have of multiplexing gain. For one video stream, the minimum bandwidth is around $70 \mathrm{Kbps}$, which is still not easy to achieve over GPRS. However future GPRS generations will be able to supply this bandwidth and more. The encouraging part is that when two or more video streams are injected, they need less bandwidth than the sum of the peak rates of each. We hope that our study triggers more investigation in the filed of multimedia over GPRS from the traffic analysis point of view. The Hurst parameter is also presented briefly.

\section{REFERENCES}

[1] Jan Beran, Robert Sherman, Murad S. Taqqu and Walter Willinger, "Long-Range Dependence in Variable-Bit-Rate Video Traffic", IEEE Transactions on Communications 43, No. 2/3/4, pp. 1566-1579, 1995.

[2] M. W. Garrett, W. Willinger, "Analysis, Modeling and Generation of Self-Similar VBR Video Traffic", Computer Communications review vol. 24, no. 4, pp. 269-280, 1997.

[3] Daniel P. Heyman and T. V. Lakshman, "What Are the Implications of Long-Range Dependence for VBR-Video Traffic Engineering?", IEEE/ACM Transactions on Networking, vol. 4, no. 3, June 1996.

[4] C. Huang, M. Devetsikiotis, I. Lambadaris and A.R. Kaye, "Modelling and Simulation of Self-Similar Variable Bit Rate Compressed Video: A Unified Approach", Proc. of the ACM Sigcomm'95, Boston, pp. 114-125, 1995.

[5] K. R. Krishnan, A.L. Neidhardt, and A. Erramilli, "Scaling Analysis in Traffic Management of Self similar Processes", ITC 15, Elsevier Science B.V., 1997.

[6] W. Leland, M. Taqqu, W. Willinger and D. Wilson, "On the Self-Similar Nature of Ethernet Traffic", IEEE/ACM Transactions on Networking vol. 2, no. 1, February 1994.

[7] Kihong Park, Gitae Kim Mark Crovella, "On the Effect of Traffic Self-similarity on Network Performance", Proceedings of the 1997 SPIE International Conference on Performance and Control of Network Systems.

[8] P. Pruthi, D Ilie, A. Popescu "Application Level Performance of Multimedia Services", SPIE International Symposium on Voice, Video, and Data Communications Boston, Sep 19-22 1999.

[9] Thierry Turletti, "H.261 Software Codec for Videoconferencing over the Internet", Rapports de Recherche, Unité de recherche no. 1834, INRIA-SOHIA Antipolis, 1993.

[10] Draft ITU-T Recommendation H.263 (1996): "Video Coding For Low Bitrate Communication". 